\title{
TWO DEFINITIONS OF AN ABELIAN GROUP BY SETS OF
}

\section{INDEPENDENT POSTULATES*}

BY

\section{EDWARD V. HUNTINGTON}

The following definitions of an Abelian (commutative) group are suggested immediately by the writer's definitions of a general group published in the Bulletin of the American Mathematical Society, ser. 2, vol. 8 (1901-1902), pp. 296-300, 388-391.†

\section{§1. First definition: by three postulates.}

A set of elements in which a rule of combination 0 is so defined as to satisfy the following three postulates shall be called an Abelian group with respect to 0 :

1) $a \circ b=b \circ a$, whenever $a, b$ and $b \circ a$ belong to the set.

2) $(a \circ b) \circ c=a \circ(b \circ c)$, whenever $a, b, c, a \circ b, b \circ c$ and $a \circ(b \circ c)$ belong to the set.

3) For every two elements $a$ and $b(a=b$ or $a \neq b)$ there is an element $x$ in the set such that $a \circ x=b$.

If we wish to distinguish between finite and infinite groups we may add a fourth postulate, either

a) The set contains $n$ elements; or

b) The set is infinite.

Familiar examples of a finite and an infinite Abelian group are the following :

A) The system of the first $n$ positive integers, with the rule of combination defined as follows:

$$
\begin{aligned}
a \circ b & =a+b & & \text { when } & & a+b \leqq n, \\
& =a+b-n & & \text { when } & & a+b>n .
\end{aligned}
$$

$B)$ The system of all integers, positive, negative and zero, with $a \circ b=a+b$; or the system of all positive rational numbers, with $a \circ b=a \times b$.

* Presented to the Society October 25, 1902. Received for publication October 4, 1902.

†Cf. E. H. Moore, Transactions, vol. 3 (1902), pp. 485-492. Professor Moore's criticism of "multiple statements" suggested the present form of postulates 1 and 2. 
The following theorems, deduced from postulates $1,2,3$, show that the present definition is equivalent to the definitions usually given.*

Theorem I. The element $x$ in 3 is uniquely determined by $a$ and $b$.

Proof. Suppose $a \circ x=b$ and also $a \circ x^{\prime}=b$; and by 3 take $\xi$ so that $x \circ \xi=x^{\prime}$. Then by hypothesis $a \circ(x \circ \xi)=b$; or, by $2,(a \circ x) \circ \xi=b$; or, $b \circ \xi=b$. Now by 3 and 1 take $\eta$ so that $\eta \circ b=x$. Then $\eta \circ(b \circ \xi)=x$; or, by $2,(\eta \circ b) \circ \xi=x$; or, $x \circ \xi=x$. Therefore $x=x^{\prime}$.

Corollary. If $a \circ b=a \circ b^{\prime}$ then $b=b^{\prime}$.

Theorem II. There is a peculiar element $e$ in the set, such that $b \circ e=b$ for every element $b$.

Proof. Take any element $a$ and by 3 take $e$ so that $a \circ e=a$; the element $e$ thus determined (Theorem I) is the peculiar element required. For, let $b$ be any other element than $a$, and by 3 and 1 take $x$ so that $x \circ a=b$. Then $x \circ(a \circ e)=b$; or, by $2,(x \circ a) \circ e=b$; or $b \circ e=b$.

Theorem III. Whenever $a$ and $b$ belong to the set, $a \circ b$ also belongs to the set.

Proof. By 3 and 1 there is an element $b^{\prime}$ such that $b^{\prime} \circ b=e$ and also an element $c$ such that $c \circ b^{\prime}=a$. Then $c=a \circ b$. For, by 3 take $\beta$ so that $a \circ \beta=c$ and $\beta^{\prime}$ so that $\beta \circ \beta^{\prime}=e$. Then

$$
c \circ b^{\prime}=a=a \circ\left(\beta \circ \beta^{\prime}\right)=(a \circ \beta) \circ \beta^{\prime}=c \circ \beta^{\prime}
$$

by 2 ; hence $b^{\prime}=\beta^{\prime}$. Then $b^{\prime} \circ \beta=\beta^{\prime} \circ \beta=e=b^{\prime} \circ b$ by 1 ; hence $\beta=b$. Therefore $a \circ b=c$.

$$
\text { Independence of postulates } 1,2,3 \text { and } a) \text {, when } n>2 \text {. }
$$

The mutual independence of postulates $1,2,3$ and $a$ ), when $n>2, \dagger$ is shown by the following systems, each of which satisfies all the other postulates but not the one for which it is numbered.

(1) The system of the first $n$ positive integers, with $a \circ b=b$.

(2) The system of the first $n$ positive integers, with the rule of combination defined as follows :

$$
\begin{aligned}
& a \circ b=a+b \quad \text { when } \quad a+b \leqq n, \\
& =a+b-n \quad \text { when } \quad a+b>n \text {; } \\
& \text { except that } a \circ b=2 \quad \text { when } a+b=n+1 \text {, } \\
& \text { and } \quad a \circ b=1 \quad \text { when } a+b=2 \text { or } n+2 \text {. }
\end{aligned}
$$

(3) The system of the first $n$ positive integers, with $a \circ b=1$.

(a) Any infinite Abelian group, such as $B$ ) above.

* The proofs of these theorems become, of course, much simpler if we confine ourselves to finite groups.

$\dagger$ When $n=1$, postulate 3 is sufficient. When $n=2$, postulates 1 and 3 are sufficient and independent. 


\section{Independence of postulates $1, \mathcal{2}, 3$ and $b)$.}

Similarly, the independence of postulates $1,2,3$ and $b$ ) is shown by the following systems :

[1] The system of all positive integers, with $a \circ b=b$.

[2] The system of all rational numbers, with $a \circ b=(a+b) / 2$.

[3] The system of all positive integers, with $a \circ b=1$.

[b] Any finite Abelian group, such as $A$ ) above.

\section{§2. SECOND DEFInition : BY FOUR POSTUlates.}

An Abelian group may be defined also by the following four postulates:

1') $a \circ b=b \circ a$, whenever $a, b, a \circ b$ and $b \circ a$ all belong to the set.

$\left.2^{\prime}\right)(a \circ b) \circ c=a \circ(b \circ c)$, whenever $a, b, c, a \circ b, b \circ c,(a \circ b) \circ c$ and $a \circ(b \circ c)$ all belong to the set.

$\left.3^{\prime}\right)$ For every two elements $a$ and $b(a=b$ or $a \neq b)$ there is an element $x^{\prime}$ in the set such that $\left(a \circ x^{\prime}\right) \circ b=b$.

4') If $a$ and $b$ belong to the set, then $a \circ b$ also belongs to the set.

To show that this second definition agrees with the first, we have only to notice that the truth of 3 follows at once from $2^{\prime}, 3^{\prime}, 4^{\prime} . \quad\left(x=x^{\prime} \circ b.\right)$

Independence of postulates $1^{\prime}, \mathscr{2}^{\prime}, \mathfrak{3}^{\prime}, \mathcal{4}^{\prime}$ and $a$ ), when $n>\mathscr{Z}$.

The independence of these postulates for finite groups, when $n>2$,* is established by the use of the following systems :

$\left(1^{\prime}\right),\left(2^{\prime}\right),\left(3^{\prime}\right),(a)$. Same as the systems (1), (2), (3), (a) above.

$\left(4^{\prime}\right)$ The system of the first $n$ positive integers, with the rule of combination defined as follows: $a \circ a=1 ; 1 \circ b=b$; otherwise $a \circ b=z$, an object not belonging to the set.

\section{Independence of postulates $1^{\prime}, \mathscr{2}^{\prime}, 3^{\prime}, 4^{\prime}$ and $b$ ).}

Similarly, the independence of these postulates for infinite groups is shown by the following systems :

$\left[1^{\prime}\right],\left[2^{\prime}\right],\left[3^{\prime}\right],[b]$. Same as the systems [1], [2], [3], [b] above.

, $\left[4^{\prime}\right]$ The system of all integers except \pm 1 , with $a \circ b=a+b$.

Harvard University, Cambridge, Mass., August, 1902.

${ }^{*}$ When $n=1$, postulate $4^{\prime}$ is sufficient. When $n=2$, postulates $1^{\prime}, 3^{\prime}, 4^{\prime}$ are sufficient and independent. 


\section{PostsCRIPT.*}

In the course of an article entitled $A$ Definition of Abstract Groups, $\dagger$ which appeared while the present paper was going through the press, Professor E. H. Moone takes up my first (three-postulate) definition of a group,

$$
\left(H_{1}\right):\left(2^{\prime}, 9^{\prime}, 10^{\prime}\right) \text {, }
$$

and after pointing out that the postulate $2^{\prime}$ can be broken up into two component statements $2_{1}^{\prime}$ and $2_{2}^{\prime}$, raises the question as to the independence of the four postulates

$$
\left(H_{1}^{\prime}\right):\left(2_{1}^{\prime}, 2_{2}^{\prime}, 9^{\prime}, 10^{\prime}\right) \text {. }
$$

As an answer to this question the following result may be not without interest: I find that either of the postulates $2_{1}^{\prime}$ and $2_{2}^{\prime}$ can be deduced as a theorem from the remaining three. That is, my first definition $\left(H_{1}\right)$ may be replaced by a new three-postulate definition, say

$$
\left(H_{1}^{\prime \prime}\right):\left(2_{2}^{\prime}, 9^{\prime}, 10^{\prime}\right) \text {, }
$$

in which the postulate $2_{2}^{\prime}$ is " milder" than the postulate 2 '. (The old proofs of independence hold for $\left(H_{1}^{\prime \prime}\right)$, for both finite and infinite groups.)

The actual deduction of $2_{1}^{\prime}$ from $9^{\prime}, 10^{\prime}$ and $2_{2}^{\prime}$ proceeds as follows : $\ddagger$ We have

$\left.9^{\prime}\right)$ For every two elements $a, b$ there is an element $x$ such that $a \circ x=b$.

$\left.10^{\prime}\right)$ For every two elements $a, b$ there is an element $y$ such that $y \circ a=b$.

$\left.2_{2}^{\prime}\right)$ If $a, b, c$ are three elements such that the products $a \circ b, b \circ c$ and $a \circ(b \circ c)$ belong to the set, then $(a \circ b) \circ c=a \circ(b \circ c)$.

Leмma. If $a \circ b=a \circ b^{\prime}$ (both products belonging to the set), then $b=b^{\prime}$.

Proof. Let $c=a \circ b=a \circ b^{\prime}$, and by. $9^{\prime}$ take $x$ so that $b \circ x=b^{\prime}$. Then, by hypothesis, $a \circ(b \circ x)=c$; or, by $2_{2}^{\prime},(a \circ b) \circ x=c$; or, $c \circ x=c$. Now by $10^{\prime}$ take $y$ so that $y \circ c=b$. Then $y \circ(c \circ x)=b$; or, by $2_{2}^{\prime},(y \circ c) \circ x=b$; or, $b \circ x=b$. Hence $b=b^{\prime}$.

TheOREM $2_{1}^{\prime}$. If $a, b, c$ are three elements such that the products $a \circ b$, $b \circ c$ and $(a \circ b) \circ c$ belong to the set, then $(a \circ b) \circ c=a \circ(b \circ c)$.

Proof. By $9^{\prime}$ take $x$ so that $a \circ x=(a \circ b) \circ c$ and also $z$ so that $b \circ z=x$. Then $a \circ(b \circ z)=(a \circ b) \circ c$. But by $2_{2}^{\prime}, a \circ(b \circ z)=(a \circ b) \circ z$. Therefore $c=z$, by the Lemma. Hence $b \circ c=x$; or, $a \circ(b \circ c)=(a \circ b) \circ c$.

In like manner we might have deduced $2_{2}^{\prime}$ from $9^{\prime}, 10^{\prime}$ and $2_{1}^{\prime}$.

HARVARD UNIVERSITY, October 31, 1902.

* Received for publication November 26, 1902.

†Transactions, vol. 3 (October, 1902), pp. 485-492.

$\ddagger$ In the case of Abelian groups this deduction is not necessary. 